\title{
Size dependent damping in picosecond dynamics of single nanomagnets
}

Aaron R. Hawkins

hawkins@ee.byu.edu

J. D. Maas

S. Wang

A. Barman

Holger Schmidt

See next page for additional authors

Follow this and additional works at: https://scholarsarchive.byu.edu/facpub

Part of the Electrical and Computer Engineering Commons

\section{Original Publication Citation}

Barman, A., S. Wang, J. Maas, A. R. Hawkins, S. Kwon, J. Bokor, A. Liddle, and H. Schmidt. "Size dependent damping in picosecond dynamics of single nanomagnets." Applied Physics Letters 9 (27)

\section{BYU ScholarsArchive Citation}

Hawkins, Aaron R.; Maas, J. D.; Wang, S.; Barman, A.; Schmidt, Holger; Kwon, Liddle A.; and Bokor, J., "Size dependent damping in picosecond dynamics of single nanomagnets" (2007). Faculty Publications. 256. https://scholarsarchive.byu.edu/facpub/256

This Peer-Reviewed Article is brought to you for free and open access by BYU ScholarsArchive. It has been accepted for inclusion in Faculty Publications by an authorized administrator of BYU ScholarsArchive. For more information, please contact ellen_amatangelo@byu.edu. 
Authors

Aaron R. Hawkins, J. D. Maas, S. Wang, A. Barman, Holger Schmidt, Liddle A. Kwon, and J. Bokor 


\title{
Size dependent damping in picosecond dynamics of single nanomagnets
}

\author{
A. Barman ${ }^{\text {a) }}$ and S. Wang \\ School of Engineering, University of California, Santa Cruz, 1156 High Street, Santa Cruz, California \\ 95064 \\ J. Maas and A. R. Hawkins \\ Department of Electrical and Computer Engineering, Brigham Young University, 459 Clyde Building, \\ Provo, Utah 84604 \\ S. Kwon, ${ }^{\text {b) }}$ J. Bokor, and A. Liddle \\ Molecular Foundry, Lawrence Berkeley National Laboratory, Berkeley, California 94720 \\ H. Schmidt ${ }^{c}$ ) \\ School of Engineering, University of California, Santa Cruz, 1156 High Street, Santa Cruz, California \\ 95064
}

(Received 7 February 2007; accepted 24 April 2007; published online 17 May 2007)

\begin{abstract}
The authors use time-resolved cavity-enhanced magneto-optical Kerr spectroscopy to study the damping of magnetization precession in individual cylindrical nickel nanomagnets. A wide range of shapes (diameters of $5 \mu \mathrm{m}-125 \mathrm{~nm}$ and aspect ratio: 0.03-1.2) is investigated. They observe a pronounced difference in damping between the micro- and nanomagnets. Microscale magnets show large damping at low bias fields, whereas nanomagnets exhibit bias field-independent damping. This behavior is explained by the interaction of in-plane and out-of-plane precession modes in microscale magnets that results in additional dissipative channels. The small and robust damping values on the nanoscale are promising for implementation of controlled precessional switching schemes in nanomagnetic devices. (C) 2007 American Institute of Physics. [DOI: 10.1063/1.2740588]
\end{abstract}

Nanomagnets will form the building blocks for magnetic data storage and future spintronic devices. Beyond data storage they have the potential to open additional avenues for medical diagnostics such as medical resonance imaging and dynamic methods for cancer treatments. ${ }^{1}$ Future technology requires faster magnetic switching in magnetic nanostructures and large-angle precessional switching has the potential to increase the operation speed to picoseconds. Two very important dynamic parameters are the precession frequency and damping. Microscale elements show complicated behavior as a result of inhomogeneous internal magnetic fields. ${ }^{2-5}$ Understanding the ultrafast dynamics of single nanomagnets is a subject of intense interest. Precessional dynamics from nanomagnet ensembles has been explored, ${ }^{6}$ albeit at the expense of dipolar broadening and dynamic dephasing effects from the neighboring elements. This problem may be overcome by combining the increased spatial sensitivity of cavity enhancement of magneto-optical Kerr effect ${ }^{7,8}$ (CE-MOKE) with the femtosecond time resolution offered by ultrafast lasers. We have recently reported the picosecond dynamics of single $\mathrm{Ni}$ nanomagnets using such an all-optical timeresolved CE-MOKE technique. ${ }^{9}$ Here we report the damping of the precessional motion of single $\mathrm{Ni}$ nanomagnets as a function of size (aspect ratio) and bias magnetic fields. Ni magnets with $150 \mathrm{~nm}$ thickness and diameters ranging between $5 \mu \mathrm{m}$ and $125 \mathrm{~nm}$ as shown in Fig. 1(a) are studied to cover a large range of aspect ratio between 0.03 and 1.2. This allows us to study damping across the transition from multidomain microscale to single-domain nanoscale magnets.

\footnotetext{
a) Also at: Department of Physics, Indian Institute of Technology Delhi, Hauz Khas, New Delhi 110016, India.

${ }^{b}$ Present address: School of Electrical Engineering, Seoul National University, Seoul 155-744, South Korea.

${ }^{c)}$ Electronic mail: hschmidt@soe.ucsc.edu
}

The Ni magnets, spaced by $5 \mu \mathrm{m}$ for optical and magnetostatic isolation, were fabricated by electron beam lithography. ${ }^{8}$ One batch of the sample was coated with a $70 \mathrm{~nm}$ silicon nitride ( $\mathrm{SiN}$ ) layer for improving the spatial sensitivity by enhancing the Kerr rotation as well as by reducing the background noise. ${ }^{8}$ The magnetic force microscope images in Fig. 1(a) show the remanent magnetic states of the Ni elements, which confirm multidomain states for larger magnets down to $250 \mathrm{~nm}$ and a single domain for the $125 \mathrm{~nm}$ magnet. The experimental setup is based on a twocolor optical pump-probe technique described in detail elsewhere. ${ }^{9}$ The sample was optically pumped ${ }^{10}$ by $15 \mathrm{~mW}$ linearly polarized $400 \mathrm{~nm}$ laser pulses of about $100 \mathrm{fs}$ pulsewidth, which induces precession in the sample. $2 \mathrm{~mW}$ lin-

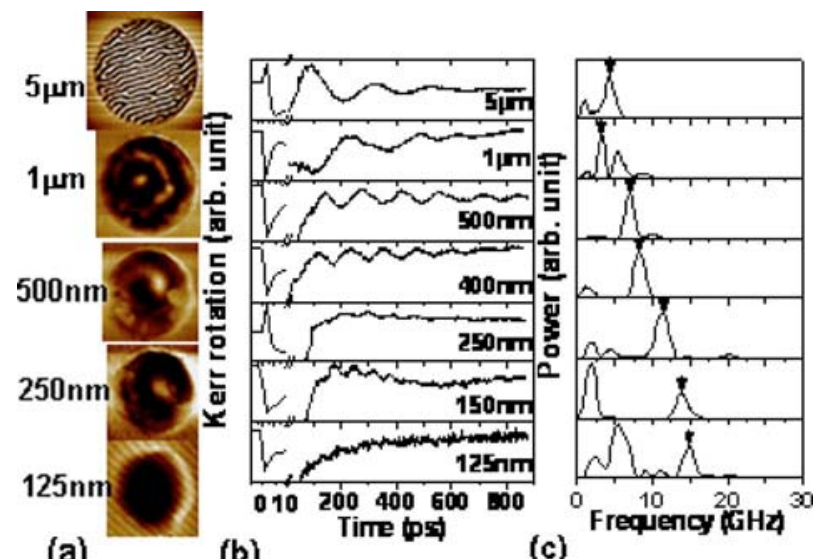

(a)

(b)

(c)

FIG. 1. (Color online) (a) Magnetic force microscope images showing the domain structures of the magnetic elements. (b) Time-resolved magnetooptical Kerr rotation (raw data) with the time scale broken between 10 and 15 ps to show the three regions of interests clearly. (c) Fast Fourier transforms of the time-resolved data after double exponential background subtraction. 
early polarized laser pulses of $800 \mathrm{~nm}$ wavelength were time delayed with respect to the pump beam to probe the dynamics by detecting polar magneto-optical Kerr rotation. A perpendicular static magnetic field up to $3.73 \mathrm{kOe}$ was applied to bias the samples during the experiment. The two-color scheme and spectral filtering before probe detection combined with the CE-MOKE provided the required sensitivity to probe single nanomagnet dynamics down to $125 \mathrm{~nm}$.

Figure 1(b) shows the time-resolved dynamics of magnets between $5 \mu \mathrm{m}$ and $125 \mathrm{~nm}$ at a bias field of $1.68 \mathrm{kOe}$. The time scale is broken between 10 and 15 ps to show three different regimes of interest clearly. Within the initial $10 \mathrm{ps}$ the fast demagnetization and the quick recovery are shown. The precession is observed after 10 ps on top of a slow exponential decay, which is subtracted from these signals and the corresponding fast Fourier transform spectra are shown in Fig. 1(c). Multiple frequency modes are observed for almost all samples where the highest frequency mode is identified as the uniform precession mode. The uniform precession mode shows a clear size dependence which has been described in detail elsewhere. ${ }^{9}$ The lower frequency mode does not show any clear size dependence, indicating different origins for magnets of varying size as discussed below.

To extract the damping of the uniform precession mode we have processed the time-resolved data by a fast Fourier transform (FFT) high-pass filtering to eliminate low frequency modes. The FFT spectra also do not show any peak above the uniform precession mode. The filtered timeresolved data possess a single uniform precession frequency and are fitted through a least square fitting routine with a damped sine function of the form

$$
M(t)=M(0) e^{-t / \tau} \sin (\omega t-\phi),
$$

where $\tau$ is the decay time of the precession defined as $\tau$ $=1 / \omega \alpha_{\text {eff }}, \omega$ is the angular frequency of the uniform precession mode given by $\omega=\gamma H_{\text {eff }}$, and $\phi$ is the initial phase of oscillation. $\alpha_{\text {eff }}$ is the "effective" damping coefficient as opposed to the intrinsic Gilbert damping, ${ }^{11} \gamma$ is the gyromagnetic ratio, and $H_{\text {eff }}$ is the effective magnetic field which includes the external bias field, demagnetizing field, volume and surface anisotropy fields, and an offset field as discussed in Ref. 9 Using the best fit values of $\omega$ (same as obtained from the FFT power spectra of time-resolved data) and $\tau$ from Eq. (1) $\alpha_{\text {eff }}$ is extracted. Since Eq. (1) is valid only in the limit of small damping $(\alpha \ll 1)$, for a further confirmation we have also extracted the damping coefficient $\left(\alpha_{\text {eff }}\right)$ from numerical solution of Landau-Lifshitz-Gilbert equation of motion $^{12}$ under macrospin model (not shown) and obtained similar results. Figure 2(a) shows the FFT filtered experimental time-resolved dynamics and the best fit curves with Eq. (1) for magnets of varying diameters at a bias field of $1.68 \mathrm{kOe}$ and Fig. 2(b) shows the extracted $\alpha_{\text {eff }}$ as a function of magnet diameter. Large qualitative and quantitative differences in $\alpha_{\text {eff }}$ between the micro- and nanoscale are found. For magnets with diameters between 5 and $3 \mu \mathrm{m} \alpha_{\text {eff }}$ reaches a maximum value of nearly 0.17 . At $2 \mu \mathrm{m}, \alpha_{\text {eff }}$ reduces sharply followed by a gradual decrease down to $500 \mathrm{~nm}$. At $500 \mathrm{~nm} \alpha_{\text {eff }}$ settles down at around 0.04, comparable to the reported damping coefficient 0.05 of continuous $\mathrm{Ni}$ thin films measured by all-optical method. ${ }^{10}$ The nearly fourfold increase in $\alpha_{\text {eff }}$ for magnets $>2 \mu \mathrm{m}$ diameter may originate from extrinsic sources such as multimagnon scattering, ${ }^{11}$ spin wave propagation for large angle Downloaded 10 Feb 2009 to 128.187.0.164. Redistribution subjec
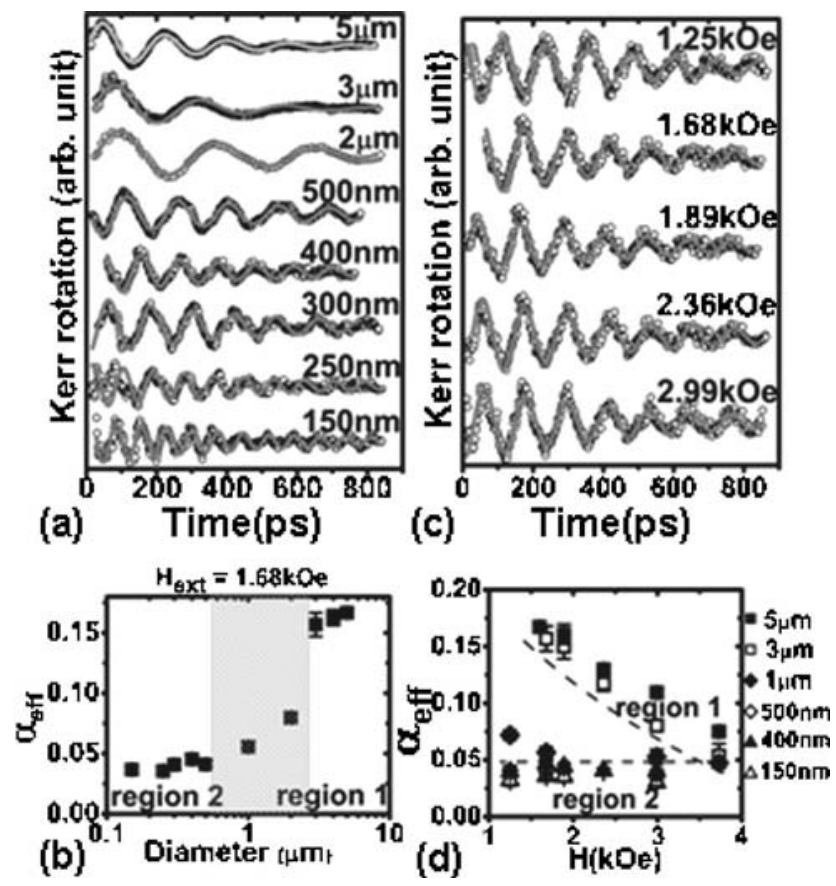

FIG. 2. (a) Experimental (open circles) and fitted (gray lines) time-resolved data from magnetic dots of varying diameter at an external bias field $=1.68 \mathrm{kOe}$. (b) The extracted effective damping coefficient $\left(\alpha_{\text {eff }}\right)$ as a function of magnet diameter. The hatched rectangle shows the transition region from a high to low $\alpha_{\text {eff. }}$ (c) Experimental (open circles) and simulated (gray lines) time-resolved data from a magnetic dot of $400 \mathrm{~nm}$ diameter at varying external bias fields. (d) The extracted $\alpha_{\text {eff }}$ for magnets with varying diameter as a function of the external bias field. A high-pass FFT filtering was applied to the experimental time-resolved data for fitting with a single damped sine function.

precession, ${ }^{13}$ spin pumping process, ${ }^{14}$ interfacial effects, ${ }^{15}$ and dephasing of incoherent spin waves. ${ }^{16}$ This size dependence of $\alpha_{\text {eff }}$ is also evident in a bias field series. Figure 2(c) shows the time-resolved experimental and fitted data for a $400 \mathrm{~nm}$ element and Fig. 2(d) shows the bias field dependence of $\alpha_{\text {eff }}$ versus magnet diameter. For magnets $>2 \mu \mathrm{m}$ a strong bias field dependence of $\alpha_{\text {eff }}$ is observed, while for magnets with diameter $<1 \mu \mathrm{m}$, no bias field dependence is observed. For magnets with intermediate diameters weak bias field dependence is observed. Even for magnets $>2 \mu \mathrm{m}, \alpha_{\text {eff }}$ approaches smaller values at larger bias fields, nearly the value obtained for the nanoscale magnets. Bias field (frequency) dependent damping has been observed in previous studies from ferromagnetic resonance linewidth ${ }^{17,18}$ measurements and an increase in damping at reduced bias field (frequency) typically has been ascribed to inhomogeneous line broadening caused by dispersion in the anisotropy field.

In order to understand the size and bias field-dependent damping behavior we investigate the frequency spectra of the experimental results carefully. In all spectra we have observed lower frequency modes of significant amplitudes with frequencies around 1-2 GHz. Figures 3(a) and 3(b) show the bias field-dependent FFT power spectra for $5 \mu \mathrm{m}$ and $400 \mathrm{~nm}$ magnets, representing regions 1 and 2 in Figs. 2(b) and 2(d), respectively. The gray solid lines show Gaussian fits to peak 1 and peak 2 . For the $5 \mu \mathrm{m}$ magnet the two resonant modes have large splitting at stronger bias fields. With reduction of the bias field, the uniform precession mode frequency decreases while the low frequency mode increases. Consequently, a significant overlap of the two modes to AlP license or copyright; see http://apl.aip.org/apl/copyright.jsp 

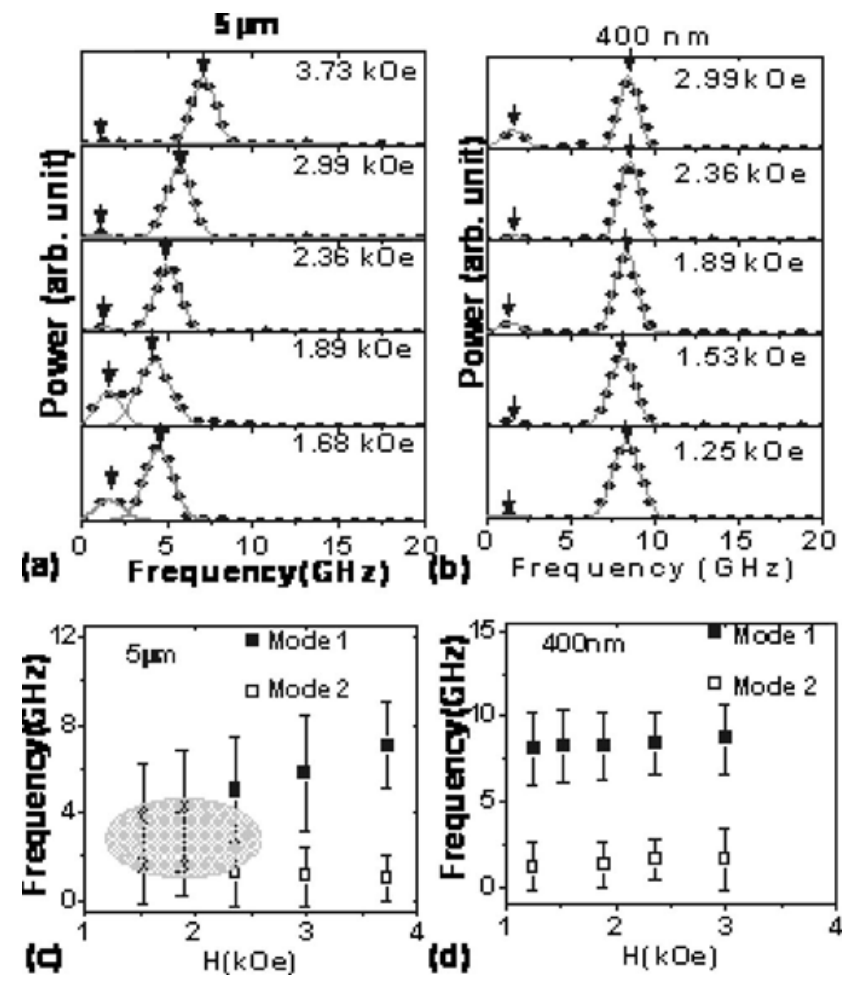

FIG. 3. FFT power spectrum of the time-resolved data (filled dots) for the (a) $5 \mu \mathrm{m}$ and (b) $400 \mathrm{~nm}$ magnets with two fitted (Gaussian) peaks as gray lines. The extracted frequencies (points) and the width of the peaks (error bars) are plotted as a function of the external bias field for the (c) $5 \mu \mathrm{m}$ and (d) $400 \mathrm{~nm}$ magnets. The hatched region in (c) shows the overlap between the two modes.

is observed from intermediate bias fields, the amount of which increases with further reduction in the bias field, shown by the hatched region in Fig. 3(c). It is the dephasing of these two modes that opens another dissipative channel and causes the enhancement of the effective damping of the uniform precession mode for samples with diameter $\geqslant 1 \mu \mathrm{m}$. In comparison, the FFT power spectra for the $400 \mathrm{~nm}$ sample also show two frequency modes but they remain well split [Fig. 3(d)] for the whole bias field range down to $1.25 \mathrm{kOe}$. The weaker bias field dependence of the uniform precession mode for nanoscale magnets due to their very large anisotropy is the main reason for this. The low frequency mode for samples $<1 \mu \mathrm{m}$ is most likely associated with the translation of the vortex core that has been predicted by static micromagnetic simulation. ${ }^{9}$ For magnets with diameter $\geqslant 1 \mu \mathrm{m}$ (where static micromagnetic simulation predicts multiple domains and significant in-plane magnetization ${ }^{9}$ ), the low frequency mode is most likely associated with the precession of an in-plane magnetization component about $H_{\text {in-plane }}$ (in-plane anisotropy field plus small in-plane component of the bias field due to the slight tilt of the sample plane with the bias field direction). The frequency of the in-plane precession mode may be expressed as 19

$$
f_{\text {in-plane }}=\frac{g \mu_{B}}{h} \sqrt{\left(H_{\text {in-plane }}\right)\left(H_{\text {in-plane }}+4 \pi M_{\text {in-plane }}\right)},
$$

where $g$ is the Lande $g$ factor, $\mu_{B}$ is the Bohr magneton, $h$ is Planck's constant, and $M_{\text {in-plane }}$ is the in-plane component of the magnetization parallel to $H_{\text {in-plane }}$. With the reduction of the out-of-plane bias field, $M_{\text {in-plane }}$ must increase in magnitude and frequency of mode 2 increases accordingly. Simultaneously, the frequency of mode 1 decreases with the reduc- tion of bias field, which results in the increased overlap of the two modes for magnets $\geqslant 1 \mu \mathrm{m}$. Other possible explanations for the observed damping behavior can be ruled out immediately including spin pumping ${ }^{14}$ and interface effects ${ }^{15}$ (due to the use of a single layer material) and multimagnon scattering ${ }^{11}$ (due to unsaturated magnetic states of samples). High-frequency spin waves may originate from nonuniform excitation along the thickness due to much shorter skin depth $(\sim 10 \mathrm{~nm})$ of the pump laser (perpendicular standing spin waves) and finite magnet size (forward volume magnetostatic modes), but are not observed in the spectra due to both smaller amplitudes and small separation from the dominant uniform mode. ${ }^{9}$

In summary, we have studied the damping of precessional motion from single nanomagnets while eliminating extrinsic ensemble effects such as dynamic dephasing ${ }^{9}$ that further complicate the dynamics. The observed damping of the uniform precession mode in microscale magnets has a large extrinsic contribution due to dephasing with a lower frequency in-plane precessional mode. On the other hand, the observation of field-independent damping for nanoscale magnets close to the thin film value ensures the reliability of coherent control of precessional switching by a straightforward pulse shaping scheme ${ }^{20}$ in nanomagnets.

The authors thank B. Hillebrands and T. J. Silva for fruitful discussions, the National Science Foundation (Grant No. ECS-0245425), and Office of Science, Office of Basic Energy Sciences, of the U.S. Department of Energy (Contract No. DE-AC02-05CH11231) for financial support.

${ }^{1}$ G. Reiss and A. Hütten, Nat. Mater. 4, 725 (2005).

${ }^{2}$ Y. Acremann, C. H. Back, M. Buess, O. Portmann, A. Vaterlaus, D. Pescia, and H. Melchior, Science 290, 492 (2000).

${ }^{3}$ J. P. Park, P. Eames, D. M. Engebretson, J. Berezovsky, and P. A. Crowell, Phys. Rev. Lett. 89, 277201 (2002).

${ }^{4}$ J. Jorzick, S. O. Demokritov, B. Hillebrands, M. Bailleul, C. Fermon, K. Y. Guslienko, A. N. Slavin, D. V. Berkov, and N. L. Gorn, Phys. Rev. Lett. 88, 047204 (2002).

${ }^{5}$ M. Belov, Z. Liu, R. D. Sydora, and M. R. Freeman, Phys. Rev. B 69, 094414 (2004).

${ }^{6}$ V. V. Kruglyak, A. Barman, R. J. Hicken, J. F. Childress, and J. A. Katine, Phys. Rev. B 71, 220409(R) (2005).

${ }^{7}$ A. V. Sokolov, Optical Properties of Metals (Blackie, London, 1967), p. 311.

${ }^{8}$ N. Qureshi, S. Wang, M. Lowther, A. R. Hawkins, S. Kwon, B. Hartleneck, and H. Schmidt, Nano Lett. 5, 1413 (2005).

${ }^{9}$ A. Barman, S. Wang, J. D. Maas, A. R. Hawkins, S. Kwon, A. Liddle, J. Bokor, and H. Schmidt, Nano Lett. 6, 2939 (2006).

${ }^{10}$ M. van Kampen, C. Jozsa, J. T. Kohlhepp, P. LeClair, L. Lagae, W. J. M. de Jonge, and B. Koopmans, Phys. Rev. Lett. 88, 227201 (2002).

${ }^{11}$ D. L. Mills and S. M. Rezende, Spin Dynamics in Confined Magnetic Structures (Springer, Heidelberg, 2003), Vol. II, p. 27.

${ }^{12}$ L. D. Landau and E. Lifshitz, Phys. Z. Sowjetunion 8, 153 (1935); T. L. Gilbert, Phys. Rev. 100, 1243 (1955).

${ }^{13}$ T. J. Silva, M. R. Pufall, and P. Kabos, J. Appl. Phys. 91, 1066 (2002).

${ }^{14}$ G. Woltersdorf, M. Buess, B. Heinrich, and C. H. Back, Phys. Rev. Lett. 95, 037401 (2005).

${ }^{15}$ R. Urban, G. Woltersdorf, and B. Heinrich, Phys. Rev. Lett. 87, 217204 (2001).

${ }^{16}$ A. Barman, V. V. Kruglyak, R. J. Hicken, J. M. Rowe, A. Kundrotaite, J. Scott, and M. Rahman, Phys. Rev. B 69, 174426 (2004).

${ }^{17}$ P. Wolf, J. Appl. Phys. 32, S95 (1961).

${ }^{18}$ Z. Celinski and B. Heinrich, J. Appl. Phys. 70, 5937 (1991).

${ }^{19}$ A. H. Morrish, The Physical Principles of Magnetism, 1st ed. (WileyIEEE, New York, 2001), p. 545.

${ }^{20}$ Th. Gerrits, H. A. M. van den Berg, J. Hohlfeld, L. Bar, and Th. Rasing, Nature (London) 418, 509 (2002). 